\title{
Novos sinônimos para espécies de SChultesia MART. E Xestaea Griseb. (GentianACEAE) ${ }^{1}$
}

\author{
Elsie Franklin Guimarães ${ }^{2}$
}

\begin{abstract}
Resumo
(Novos sinônimos para espécies deSchultesia Mart. e Xestaea Griseb. (Gentianaceae)). Foram sinonimizados seis táxons (Schultesia apiculata Huber, S. pallens Bunbury, S. stenophylla y pallens (Bunbury) Progel, S. guianensis f. lutescens Standl. \& Steyerm., S. hassleriana Chodat, S. brachyptera Cham. f. heterophylla (Miq.) Jonker) em espécies do gêneroSchultesia e dois (Schultesia lisianthoides (Griseb.) Benth. \& Hook. ex Hemsl., S. peckiana B.L. Rob.) em uma espécie de Xestaea (Gentianaceae).

Palavras-chave: Taxonomia, Gentianaceae, Schultesia, Xestaea, novos sinônimos.
\end{abstract}

\section{Abstract}

(New synonyms for Schultesia Mart. and Xestaea Griseb. species (Gentianaceae)). This work proposes six synonyms (Schultesia apiculata Huber, S. pallens Bunbury, S. stenophylla r pallens (Bunbury) Progel, $S$. guianensis f. lutescens Standl. \& Steyerm., S. hassleriana Chodat, S. brachyptera Cham. f. heterophylla (Miq.) Jonker) in species of the genus Schultesia Mart. and two (Schultesia lisianthoides (Griseb.) Benth. \& Hook. ex Hemsl., S. peckiana B.L. Rob.) in species of Xestaea Griseb. (Gentianaceae).

Key-words: Taxonomy, Gentianaceae, Schultesia, Xestaea, new synonyms.

\section{INTRODUÇÃo}

Este trabalho é parte dos resultados obtidos na revisão taxonômica do gênero Schultesia (Guimarães 2002). O gênero foi descrito por Martius em 1827. Desde então, diversos autores deram suas contribuições, tendo se destacado os estudos realizados por Grisebach (1836), Progel (1865) e Gilg (1895), que procuraram reunir as espécies, elaborando chaves analíticas ou estabelecendo sistemas de classificação. $O$ gênero apresenta distribuição pantropical, com centro de diversidade na América do Sul, principalmente no Brasil, onde ocorrem 18 espécies.

O gênero monotípico Xestaea, com distribuição na América Central e norte da América do Sul, foi incluído emSchultesia por Gilg (1865). Atualmente, Struwe \& Albert(2002), com base nos estudos em biologia molecular e morfologia, e nos resultados obtidos por Maguire \& Boom (1989) e Nilsson (2002) para o pólen de Xestaea, restabelecem o gênero de Grisebach, considerando que o padrão da exina é claramente diferente de Schultesia.
Concorda-se com os autores no que diz respeito ao restabelecimento do gênero Xestaea.

Propõe-se, neste trabalho, novos sinônimos para espécies dos dois gêneros, que habitam geralmente brejo herbáceo, campo rupestre, cerrado, veredas, savanas e, às vezes, áreas antrópicas.

\section{Material e Métodos}

Foram consultados materiais botânicos herborizados, depositados nos herbários $\mathrm{B}, \mathrm{BR}$, CGE, F, G, GH, K, M, MG, MO, RB, S, U (siglas conforme Holmgren et al. 1990), e materiais oriundos de diversas coletas realizadas no período de 1999 a 2002.

\section{Resultados e Discussão}

Schultesia brachyptera Cham., Linnaea 8: 8. 1833. Grisebach, Gen. Sp. Gentianarum, 128. 1839; Grisebach in A. De Candolle, Prodr. 9: 68. 1845; Progel in Martius, Fl. bras. 6(1): 208, fig. 57. 1865; Jonker in Pulle, Fl. Suriname 4(1): 402. 1936; Standley \& Williams, Fieldiana (Bot.) 24(8): 328. 1969; Lemeé, Fl. Guyane

Artigo recebido em 11/2003. Aceito para publicação em 06/2004.

${ }^{1}$ Este trabalho é parte da tese apresentada no Museu Nacional da Universidade Federal do Rio de Janeiro

${ }^{2}$ Pesquisadora do Instituto de Pesquisas Jardim Botânico do Rio de Janeiro / Bolsista do CNPq. Rua Pacheco Leão, 915 22460-030 - Rio de Janeiro - RJ. eguimar@jbrj.gov.br 
Française 3: 278. 1953; Maguire \& Boom, Mem. New York Bot. Gard. 51: 18. 1989. Typus: E Bahia omnium Sanctorum Brasilia misit Lhotzky. (typus não localizado).

Schultesia apiculata Huber, Bol. Mus. Paraense Hist. Nat. 2(2): 511. 1898. Typus: Ad fluvium Maraca Guianae Brasiliensis. VII.1896, M. Guedes $n^{\circ} 623$. (holotypus MG!; isotypus RB!). Syn. nov.

Chamisso (1833) descreve $S$. brachyptera detalhadamente, a partir do material coletado por Lhotzsky no estado da Bahia, mencionando que as folhas inferiores são elípticas, as superiores linear-lanceoladas, estreitas, agudas, com cálice membranáceo com ângulos providos de nervuras espessas e alas estreitas desprovidas de nervuras. Flores purpúreas ou violáceas; óvulos numerosos.

Grisebach (1839) trata desta espécie, mencionando que as flores possuem pedicelos muito curtos, que as folhas inferiores são distintas das superiores, obtusas e acuminadas, respectivamente, e que o cálice apresenta ala linear. Cita como material estudado os exemplares de Sellow (Brasilia), Salzmann (Bahia), Leprieur s.n. e Blanchet $\mathrm{n}^{\mathrm{0}} 72$.

Progel (1865) situa $S$. brachyptera entre as espécies com cálice carenado ou alado, sendo que as alas são desprovidas de nervuras. Cita todos os materiais mencionados pelos autores anteriores, acrescenta os de Hostmann 427, Splitgerber e Wullschlägel, sem número. Huber (1898) descreve Schultesia apiculata com base em material coletado no Rio Maracá sob o $\mathrm{n}^{\mathrm{o}} 623$, com holotypus depositado no herbário do Museu Paraense Emílio Goeldi (MG); e Guimarães (1966) cita o isotypus para o herbário do Jardim Botânico do Rio de Janeiro (RB).

A análise das coleções permitiu colocar a espécie de Huber como sinônimo de $S$. brachyptera, principalmente por apresentar o cálice carenado, o que concorda com aqueles das coleções dos materiais analisados e com a estampa fornecida por Progel (1865, fig. 57).

Embora não se tenha recebido o material tipo coletado por Lhotzsky na Bahia, foram examinados todos os exemplares acima citados, o que deu-nos uma convicção quando comparados com a diagnose original. Além disso, Progel menciona o typus colocando-o junto aos demais materiais por ele e por nós também estudados, apresenta estampa das peças florais que concordam com nossa conceituação, principalmente quando apresenta o detalhe do cálice desprovido de alas nervadas.

Por outro lado, em correspondência mantida com o pesquisador P. Maas, da Universiteit Utrecht, quando da consulta da localização do typus do material de Lhotsky, o mesmo não conseguiu encontrar o referido material, que ainda se pretende localizar.

Schultesia gracilis Mart., Nov. Gen. et Sp. 2(2): 105, tab. 181. 1827. G. Don, Gen. hist. 4(1): 196. 1838; Grisebach, Gen. Sp. Gent. 128. 1839; Progel in Martius, Fl. bras. 6(1): 206. 1865; Cordeiro in Giuliettiet al., Bol. Bot. Univ. São Paulo 9: 237. 1987. Typi: Crescit in campis montanis inter Lorena Villam et $S$. Pauli Civitatem variis locis, in Provincia $S$. Pauli, prope S. João d'El Rey, Villa Rica et alibi in Provincia Minarum, altitudine 15003900 pedum supra oceanum, Martius syntypi (M); Prov. Minas Gerais, Habitat in campis Contenda - lectotypus (M!) hic designatus.

Schultesia pallens Bunbury, Proc. Linn. Soc. London 1: 110. 1849; Malme, Ark. Bot. 3(12): 11. 1904. Typus: Prope Gongo Soco in Prov. Minas Geraes (typus não localizado). Syn. nov.

Schultesia stenophylla $\gamma$ pallens (Bunbury) Progel, 1.c.: 207; Malme, 1.c.: 10. 1904. Typus: Prope Gongo Soco in Prov. Minas Geraes (typus não localizado). Syn. nov.

Martius (1827) descreve com detalhes S. gracilis e apresenta estampa elucidativa com base em material coletado em São Paulo, Lorena e várias outras localidades daquele estado; em "São João d'El Rey, Villa Rica" como também em outras localidades do estado de Minas Gerais, sem, no entanto, especificálas. Embora não se tenha recebido alguns dos 
exemplares com localidade mencionadas por Martius em sua diagnose original, recebemos outros por ele coletados e por nós examinados, oriundos do herbário de Munique (M), que nos permitiu considerá-los como sintypi da coleção, "Habitat in campis ad Contenda Prov. Min. Ger. Martius Iter Brás - Apr.”, Prov. Minas Gerais, Martius Iter Brasil, (963) e Prov. Minas Gerais, Martius (560), os três exemplares determinados por Martius como $S$. gracilis Mart.

Quando da análise dos referidos exemplares considerados sintypi, selecionouse o exemplar "Prov. Minas Gerais, Habitat in campis Contenda" como o lectotypus de Schultesia gracilis Mart., uma vez que esta exsicata detém uma amostra que se mostrou idêntica, quando comparada com o desenho original de Martius.

Malme (1904), com base nos estudos dos materiais de Regnell III-896 e III-1788 depositados no herbário de Stockholm (S), faz algumas referências sobre S. pallens, indicando ser esta espécie provável sinônimo de S. gracilis.

Com base na diagnose de Bunbury (1849), que menciona ser esta espécie caracterizada por apresentar "foliis ovatis ellipticisque acutiusculis: summis lineari-lanceolatis acuminatis, floribus terminalibus subsolitariis, alis calycis dilatatis semiovatis, corollae laciniis obovato-rhombeis breviter acuminatis integerrimis", concorda-se com Malme (1904), dado que foram analisados os mesmos exemplares por ele estudados.

As pesquisas realizadas nos herbários para a localização da coleção-tipo de $S$. pallens foram infrutíferas, mesmo quando consultado o herbário da Universidade de Cambridge (CGE), onde se supõe esteja depositada a coleção de C. J. F. Bunbury (Urban 1906).

Indica-se $S$. pallens como sinônimo novo, levando-se em consideração as informações de Malme, com as quais concorda-se, e, sobretudo, as observações das características similares entre esta espécie e $S$. gracilis.
Schultesia guianensis (Aubl.) Malme var. guianensis, Ark. Bot. 3(12): 9. 1904. Lemeé, Fl. Guyane Française 3: 278. 1953; Standley \& Williams, Fieldiana (Bot.) 24(8): 330. 1969; Elias \& Robyns in Woodson, Schery et al. Fl. Panamá. Ann. Missouri Bot. Gard. 62: 82. 1975; Maguire \& Boom, Mem. New York Bot. Gard. 51: 19. 1989.

Exacum guianensis Aubl., Hist. P1. Guiane 1: 68, tab. 26, fig. 1. 1775. Typus: Guiane Française. Coleção de Aublet: P-R3: 113 (holotypus - P apud Howard (1983)).

Schultesia guianensis f. lutescens Standl. \& Steyerm., Field. Mus. Nat. Hist., Bot. Ser. 23(2): 77. 1944. Typus: Guatemala, Dept. Jalapa, damp meadow in oak forest, near Jalapa, alt. 1360 meters, november 1940, Paul C. Standley 76561 (holotypus F!); Honduras, Dept. Comayagua, dry open bank, near Siguatepeque, 1400 meters, February 1928, P.C. Standley 56088 (paratypus F!). Syn. nov. Standley \& Steyermark (1944) descrevem S. guianensis f. lutescens, com base em material coletado na Guatemala, em Jalapa. Esclarecem que esta forma difere da típica pela corola pálido-amarelada ou brancoamarelada ou por ser ainda erva de pequeno porte com cerca de $2-3 \mathrm{~cm}$ de altura. A análise do holotypus (P. C. Standley 76561) e do paratypus (P. C. Standley 56088), depositados no Field Museum of Natural History (F), o primeiro em flor e o segundo em fruto, permitiu estabelecer a sinonimia para $S$. guianensis.

Schultesia heterophylla Miq., Linnaea 19: 137. 1847. Walpers, Repert. Bot. Syst. 6: 502. 1847; Grisebach, Fl. Brit. W. I. 5: 423. 1862; Progel in Martius, Fl. bras. 6(1): 208. 1865; Hemsley, Biol. cent. - amer., Bot. 2(11): 348. 1882; Urban, Symb. antill. 4(3): 490. 1910; Urban, 1.c. 8(2): 537. 1921; Sauget \& Liogier, Contr. Ocas. Mus. Hist. Nat. Colegio "De La Salle" 4: 164. 1957; Elias \& Robyns in Woodson \& Schery, Ann. Missouri Bot. Gard. 62: 81. 1975. Typus: Crescit prope plantationes Vier Kinderen et l'Inquietude. m. Sept., Focke s.n., apud Jonker (1936), (typus não localizado). 
Schultesia hassleriana Chodat, Bull. Herb. Boissier 3, ser. 2: 549. 1903. Typus: Ad marginem lagunae pr. Arroyo Primero (Apa), Febr., E. Hassler 8495 (holotypus G; isotypi $\mathrm{B}$ !; K!; MO!; Foto do typus F!). Syn. nov.

Schultesia brachyptera f. heterophylla (Miq.) Jonker in Pulle, Fl. Suriname 4(1): 403. 1936. Typus: Crescit prope plantationes Vier Kinderen et l' Inquietude. m. Sept., Focke s.n., apud Jonker (1936), (typus não localizado). Syn. nov.

Miquel (1847) classifica S. heterophylla com base em material das "Plantae fockeanae" "Crescit prope plantationes Vier Kinderen et l'Inquietude, M. Sept.". Esclarece que é espécie de porte maior que $S$. brachyptera, e que apresenta alas do cálice espessas nas margens, levemente denticuladas.

Chodat (1903) descreveu S. hassleriana, tomando como referência o material coletado em "ad marginem lagunae" pr. Arroyo Primeiro (APA) feb. Hassler 8495, com holotypus depositado no herbário de Genève $(\mathrm{G})$, tendo-se examinado deste os isotypi depositados nos herbários de Berlim (B), Kew (K) e Missouri Botanical Garden (MO).

Jonker (1936) trata S. heterophylla como forma de $S$. brachyptera. Assinala em seu trabalho o material coletado por Focke s.n., como correspondente à forma heterophylla, entre outros considerados por ele como da típica.

Não recebemos o material coletado por Focke na coleção examinada de Utrecht (U) e, conforme P. Maas (com. pess.), o mesmo não existe naquele herbário. Também não foi encontrado nos demais para os quais solicitouse empréstimo de material para os estudos.

Xestaea lisianthoides Griseb., Linnaea 22: 36.1849 .

Schultesia lisianthoides (Griseb.) Benth. \& Hook. ex Hemsl., Biol. cent. - amer., Bot. 2(11): 348. 1882. Standley \& Williams, Fieldiana (Bot.) 24(8): 331. 1969; Elias \&
Robyns, in Woodson \& Schery, Fl. Panamá. Ann. Missouri Bot. Gard. 62: 83, fig. 7. 1975. Typus: Ad fossas humidas pr. Bituco fl. $\mathrm{m}$. febr., E. Otto 553 (holotypus BR!). Syn. nov. Schultesia peckiana B.L. Rob., Proc. Amer. Acad. 45: 399. 1910. Typus: British Honduras about plantations and in the openings of the forests nest Manatee Lagoon, 27 january, 1906 Reof. Norton E. Peck 318 (holotypus GH!; Foto do typus F!). Syn. nov.

Grisebach (1849) descreve $X$. lisianthoides com base em material coletado na Venezuela "ad fossas humidas pr. Bituco, fl. m. febr., E. Otto 553", depositado em Bruxelles; mais tarde Bentham \& Hooker (1876) colocam o gênero Xestaea como sinônimo de Schultesia.

\section{Agradecimentos}

Ao Dr. Jorge Fontella Pereira, pelo apoio, incentivo e colaboração na discussão do tema. Aos curadores dos herbários, pelo empréstimo dos materiais. À Ms. Mariana Machado Saavedra, pela atenção que sempre dispensou a esta pesquisadora. Ao Conselho Nacional de Desenvolvimento Científico e Tecnológico - CNPq, pela bolsa concedida.

\section{REFERÊNCIAS BIBLIOGRÁFICAS}

Aublet, J. B. C. F. 1775. Histoire des Plantes de la Guiane Françoise. Londres, Paris (Pierre-François Didot jeune), vol. 1, p. 68-70, tab. 26, fig.1.

Bentham, G. \& Hooker, J. D. 1876. Gentianeae. In: Bentham, G. \& Hooker, J. D. Genera Plantarum. London, Lovell Reeve e Co., v. 2, part. 2, p. 799-820.

Bunbury, C. J. F. 1849. Remarks on some new or rare species of Brazilian Plants. Proceedings of the Linnean Society of London 1: 101-113.

Chamisso, L. A. von. 1833. Spicigeluim plantarum e families jam prius rcensites praesertim brasiliensium serius a Sellowio missarum. Gentianeae. Linnaea 8: 7-17. 
Chodat, R. 1903. Gentianaceae. In: Chodat, R. \& Hassler, E. Plantae Hasslerianae. Bulletin de 1'Herbier Boissier, ser. 2, 3: 532-552.

Cordeiro, I. 1987. Gentianaceae. In: Giulietti A. M. \& al. (eds.). Flora da Serra do Cipó, Minas Gerais. Boletim de Botânica da Universidade de São Paulo 9: 227-242.

Don, G. 1838. Gentianeae. In: Don, G. A general history of the dichlamydeous plants. London (J. G. and F. Rivington), v. 4, part 1, p. 173-214.

Elias, T. S. \& Robyns, A. 1975. Gentianaceae. In: Woodson, R. E. Jr., Schery, R. W. et al. Flora of Panama. Annals of the Missouri Botanical Garden 62: 61-101.

Gilg, E. 1895. Gentianaceae. In: Engler, H. G. A. \& Prantl, K. A. E (eds.). Die Natürlichen Pflanzenfamilien..., Leipzig (Wilhelm Engelmann) v.4, abt. 2, p. 50108, fig. 29-48.

Grisebach, A. H. R. 1836. Observationes quaedam de Gentianearum familiae characteribus. Dissertatio, ... Berlin (Nietack), $37 \mathrm{p}$.

Grisebach, A. H. R. 1839. Genera et species Gentianearum,... Stuttgart, Tübingen (Cotta), $364 \mathrm{p}$.

1845. Gentianaceae. In: Candolle, A. L. P. P. de (Ed.). Prodromus systematis naturallis regni vegetabilis... Paris (Treuttel et Würtz), Strasbourg, London, v. 9, p. 38-141.

1849. Gentianeae Juss. In: J. F.

Klotzsch. Beiträgen zu einer Flora der Aequinoctial-Gegenden dr neuen Welt. Linnaea 22: 32-46.

1862. Gentianeae. In: Grisebach, A. H. R. Flora of the British West Indian Islands. London (Lovell Reeve e Co.), part 5, p. 411-506.

Guimarães, E.F. 1966. Gentianaceae do estado da Guanabara. Rodriguésia 25(37): 29-35.

Guimarães, E. F. 2002. Schultesia Mart. (Gentianaceae) - Revisão taxonômica. Tese de doutorado, Rio de Janeiro,
Universidade Federal do Rio de Janeiro / Museu Nacional, 356p.

Hemsley, W. B. 1882. Gentianeae. In: Hemsley, W. B. (ed.). Biologia centraliamericana or Contributions to the knowledge of the fauna and flora of Mexico and Central America. Botany... London, R. H. Porter... and Dulau \& Co., vol. 2, part. 11, p. 344-353.

Holmgren, P. K.; Holmgren, N. H. \& Barnett, L. C. 1990. Index Herbariorum. Part. 1: The Herbario of the world. Regnum vegetabile. New York Botanical Garden, New York, $8^{a}$ ed., 693 p.

Howard, R. A. 1983. The plates of Aublet's Histoire des plantes de la Guiana Françoise. Journal of the Arnold Arboretum 64: 255-292.

Huber, J. E. 1898. Materiaes para a Flora Amazonica. Boletim do Museu Paraense de História Natural e Ethnographia 2(2): 496-514.

Jonker, F. P. 1936. Gentianaceae. In: Pulle, A. A. (ed.). Flora of Suriname... Amsterdam, Koninklijke Vereeniging Indisch Instituut, v. 4, part.1, p. 400-427.

Lemée, A. M. V. 1953. Gentianacées. In: Lechavalier, P. (ed.). Flore de la Guyane française. Paris, vol. 3, p. 277-288.

Maguire, B. \& Boom, B. M. 1989. Gentianaceae (Part 3). In: Maguire, B. et al. (eds.). The Botany of the Guyana Highland - Part. XIII. Memoirs of the New York Botanical Garden 51: 1-56.

Malme, G. O. 1904. Die Gentianaceen der Zweiten Regnell'schen Reise. Arkiv för Botanik utgivet av k. svenska vetenskapsakademien 3(12): 1-23, pl. 1-2.

Martius, C. F. P. von. 1827. Nova genera et species Plantarum..., Monachii (München), vol. 2, part. 2, p. 69-148, pls. 161-200.

Miquel, F. A. W. 1847. Plantae Fockeanae in Symbolae ad floram surinamensem. Linnaea 19(2): 129-145.

Nilsson, S. 2002. Gentianaceae: a review of palinology. In: Struwe, L. \& Albert, V. A. 
(eds.), Gentianaceae: systematics and natural history. Cambridge: Cambridge University Press, p. 377-497.

Progel, A. 1865. Gentianaceae. In: Martius, C. F. P. von, Eichler A. W. \& Urban, I. (eds.). Flora Brasiliensis... München, Wien, Leipzig, v. 6, part. 1, p. 197-248, pl. 55-66.

Robinson, B. L. 1910. Spermatophytes new or reclassified, chiefly Rubiaceae and Gentianaceae. Proceedings of the American Academy of Arts and Sciences 45(17): 399-400.

Sauget, J. S. \& Liogier, E. E. 1957. Gentianaceae. In: Flora de Cuba. Contribuciones Ocasionales del Museo de Historia Natural del Colegio "De La Salle" 4(16): 158-170.

Standley, P. C. \& Steyermark, J. A. 1944. Studies of Central American Plants IV. Field Museum of Natural History. Botanical Series 23(2): 77.

Standley, P. C. \& Williams, L. O. 1969. Gentianaceae. In: Standley, P. C. \& Williams, L. O. Flora of Guatemala. Fieldiana: Botany 24(8): 328-331.

Struwe, L. \& Albert, V. A. 2002. Gentianaceae: systematics and natural history. Cambridge: Cambridge University Press, 652p.

Urban, I. 1906. Vitae itineraque collectorum botanicorum, notae collaboratorum biographicae. In: Martius, C. F. P. von, Eichler A. W. \& Urban, I. (eds.). Flora brasiliensis... München, Wien, Leipzig, v. 1, part. 1, p. 1-268.

Urban, I. 1910. Gentianaceae. In: Urban, I. (ed.), Symbolae Antillanae, Berolini [Berlin] (frates Borntraeger), Parisiis (Paul Klincksieck), Londini (Williams \& Norgate), vol. 4, part. 3, p.353-528.

Urban, I. 1921. Gentianaceae. In: Urban, I. (ed.), Symbolae Antillanae, Berolini [Berlin] (frates Borntraeger), Parisiis (Paul Klincksieck), Londini (Williams \& Norgate), vol. 8, part. 2, p.481-860.
Walpers, G. G. 1847. Gentianaceae. In: Walpers, G. G. Repertorium Botanices Systematicae. Tomus VI. Lipsiae sumtibus Fredrici Hofmeister, p. 499-510. 